\title{
IAU Symposium 317 Summary
}

\section{Raffaele G. Gratton}

INAF-Osservatorio Astronomico di Padova

email: raffaele.gratton@oapd.inaf.it

\begin{abstract}
The assembly of the halo yields fundamental information on the formation and evolution of galaxies: this was quite exhaustively discussed at this very important symposium. I present a brief personal summary of the meeting, outlining those points that I found more exciting and suggestive. I also remarked a few areas that were possibly not enough expanded. I found this research field extremely interesting and I think there are great expectations for new developments in the next few years, thanks to the new large spectroscopic surveys and the ESA GAIA satellite.
\end{abstract}

Keywords. Galaxy (the): halo

\section{Introduction}

First of all: it is an honour and also a very difficult task to summarize such a lively and wide meeting. Giving justice to all speakers is impossible, and I am certainly not able to give a single coherent speech that combines all have been said by many of you, often much more competent than me. In addition, such a coherent speech have already been given by our chairman, O. Gerhard, in his very nice general talk. In this summary to the meeting I will then present my personal view, trying to summarize what I have learnt in these days and those points that are not yet entirely clear to me.

The first thing I learnt is that there is a quite uniform approach within the field. For instance, all the simulations and interpretations presented at the meeting are based on the paradigm of hierarchical growth of galaxies in a $\Lambda$-CDM universe. While this might be perfectly reasonable, I think it should perhaps not be considered as obvious as it apparently seems to be. In general, I would encourage people to discuss this approach more in depth, and I would welcome and consider possibly more enriching a less uniform approach, though I understand it may be dangerous especially for young investigators to lose time following routes that may reveal sterile.

The content of my talk is the following: (i) Stellar halo structure; (ii) First stars; (iii) Globular clusters; (iv) Dwarf spheroidals; and (v) What is next?

\section{Stellar halo structure}

Many talks considered this issue, that was central to the meeting. Different tracers of the halo were considered, including red giant branch stars (A. McConnachie, M. Rejkuba, M. Steinmetz, D. Crnojevic, M. Hilker, K. Gilbert, A. Kupper, S. Koposov, E. Peng, M. Collins, J. Greene, A. Ferguson, A. Monachesi, R. de Jong, M.G. Lee, C. Grillmair, A. Sheffield), globular clusters (GCs: S. Larsen, M. Hilker, J. Brodie), planetary nebulae (PNe: M. Arnaboldi, W. Reid, J. Brodie, N. Napolitano), and RR Lyrae variables (G. Fiorentino. J. Cohen, P. Pietrukowicz). There were very interesting reviews and contributions about models (K. Johnston, J. Read, B. Cook, A. Helmi, M. Hirschmann, G. Hensler), while the talk by Jorge Penarrubia was more on methodology.

In general, a quite coherent pattern emerges from all this work. K. Johnston spoke about the origin of the halo stars and considered three main components: accreted, in 
situ, and kicked out from disk. This general view was shared by all speakers. The relative fractions of halo stars that are due to these different mechanisms depend on distance from the center. The in situ component prevails in the inner halo while the accreted one in the outer halo. Stars that originated in the disk and kicked out into the halo makes up some $1-30 \%$ of the total within $\sim 20 \mathrm{kpc}$ (Zolotov et al. 2009, 2010, Tissera et al. 2012, 2013 etc). My comment here is that this view considers two main regions in the halo: inner and outer halo. Given the strong galactocentric density gradient existing in the halo, the inner halo is the vast majority of the halo. On the other hand, the outer halo has less dynamical evolution and better conserved traces of its origin. Likely for this reason, most of this meeting focused on the outer halo. A second issue concerns the nature of the accretion episodes, and in particular the distinction between dry and gas-rich mergers. Most theoretical work concerns dry mergers, likely because they are simpler to model. However, gas-rich mergers may imply much larger accreted masses, and might be more relevant to understand the formation of the bulk of the halo.

I have learnt a number of simple rules: (i) Since there is a Mass (actually Luminosity) Metallicity relation for dwarf galaxies (Kirby et al. 2013), then low metallicity stars are mainly produced by minor mergers while high metallicity ones are produced by major mergers (B. Cook talk). (ii) Thin/cold streams are related to GCs (C. Grillmair talk). (iii) The offset between leading/trailing tidal tails is determined by the position of the Lagrangian points (A. Kupper talk). (iv) Make often back ups of my notes. Surely I should have learnt also other things but likely I was not good enough to do so.

At this meeting, we have seen fantastic new results concerning the halo of the Milky Way and of M31: this shows that the research field is extremely active. Many new dwarf Spheroidals (dSph) are being discovered; the total of such objects known in the Milky Way is over 40 now. This increment might suggest revisiting of the missing satellite issue: is it really still a problem? The 40 faint satellites of the Milky Way discovered so far are consistent with the number of objects predicted from the SDSS brighter limit (Tollerud et al. 2008, ApJ 688, 277). Then there is still a missing satellite objects for the Milky Way. On the other hand, I notice that most new dSphs are very small while the problem of the missing satellites appears at rather large masses $\left(\mathrm{M}>10^{8} M_{\odot}\right)$. Then, a more attractive possible explanation of the missing satellite issue is on my opinion related to the issue of the mass distribution in dSph's, that is the question of cuspy vs core. In fact, as outlined in several talks, cores are more easily destroyed than cusps by tides during the dynamical interaction with the Milky Way. It is then possible that many satellites of the Milky Way (and of other galaxies too) are destroyed during the violent phases of the dynamical interaction.

Several studies support the view that the accretion history of the Milky Way was different from that of M31: the latter has more prominent substructures, and much more recent accretions. This shows that there is a considerable variance in accretion histories. This also results from simulations of the formation of stellar halos. This is a strong motivation to study (lots of) galaxy halos because our own is not enough and representative. The study of external halos is however difficult because it requires deep imaging to very low surface brightness and/or resolved populations.

If we now look more in detail to the Milky Way, talks in the meeting outlined the following facts. The census of surviving satellites is still incomplete. From existent data, the predicted number of satellites is between 50 to 500 within a galactocentric distance $R_{\mathrm{G}}$ of $R_{\mathrm{G}}<280 \mathrm{kpc}$, the exact number depending on the assumed density profile. The density profile of halo stars from various tracers show a steepening at $R_{\mathrm{G}} \sim 25 \mathrm{kpc}$. The velocity dispersion of halo stars may be obtained from data obtained by the SDSS: the velocity dispersion is independent of distance for $R_{\mathrm{G}}<10 \mathrm{kpc}$ showing that the inner 
halo is well mixed. There are few information for large distances, though in some cases we have data reaching as far as $150 \mathrm{kpc}$. This data suggests that the profile flattens at distances $>100 \mathrm{kpc}$. There are four main substructures: the Sagittarius dSph and the related stream; the Monoceros ring; the Virgo cloud; and the Hercules-Aquila cloud. Summing up all these main substructures make $\sim 210^{8} M_{\odot}$ in stars and probably about 20 GCs. Sagittarius dSph alone provides about $20 \%$ of halo debris. From the halo there is no evidence for late accretion of large satellites in the Milky Way (Justin Read). I notice that this was also derived from the characteristics of the thin disk, that would have been significantly heated by such an event.

At variance with the Milky Way, M31 is a good example of disk heating with a substantial fraction of the halo being attributed to the kicked out component. Johnston noticed that there is plenty of substructures around the disk. McConnachie showed wonderful images from the PAndAS survey that display clear structures over smooth halo (Ibata et al. 2014): he reported that only a small fraction of the M31 halo appears smooth; the smooth fraction is about $6 \%$ at high metallicity and $60 \%$ at low metallicity. In any case, it is clear that the halo of M31 is very extended and more metal-rich than that of the Milky Way. Ferguson noticed that most of the substructures in M31 are metal-rich $([\mathrm{Fe} / \mathrm{H}]>-1.1)$ and only a tiny fraction has dSph metallicity. Possibly the most striking structure seen in the M31 halo is the giant stream extending toward M33 that has a total luminosity $M_{V}$ brighter than -14 , may be $M_{V}=-16$. This can be modelled as a satellite of $10^{9} M_{\odot}$ falling on a radial orbit $\sim 1$ Gyr ago (A. Ferguson)

There are possibly puzzling results concerning the M31 halo. Mackey et al. (2010) used data from the PAndAS survey to show that the GCs in M31 align with streams in halo. Outer halo GCs show net rotation, apparently aligned with the disk rotation: is this only a coincidence? In her talk Helmi discussed this point and suggested that it is possibly explained by a peculiar point of view. I think this issue requires further investigation.

Several talks discussed the structure of the halos of galaxies in the Virgo cluster. It is found that large ellipticals at a redshift of $z \sim 2$ are 3-4 times more compact than at $z=0$; hence most of the halo has been accreted later. An important issue concerns the relation between the halos of large galaxies and the intracluster light (ICL: see talks by C. Mihos and M. Arnaboldi). The overall idea is that gravitational interaction in groups strips stars and feed intracluster light/halo. According to this scenario, the ICL grows with time, making up to $10-20 \%$ of cluster light at $z=0$. The Virgo cluster is littered with signatures of all the events that give rise to ICL. An important question is if there is a dominant formation channel for ICL. Studies of the stellar population in the Virgo ICL shows that it is mostly composed of old stars with a considerable range in metallicity. For various reasons (consideration of cosmic variance and attempt to reconstruct the evolution of ICL in a statistical way) there is need to get information on farther systems. Unfortunately, at such large distances we lose the possibility to study discrete populations. In general, a major is issue is how to separate halo from ICL, and even if it is reasonable to do this.

\section{First stars}

First stars are very interesting for various reasons. Among others, I may cite their possible relevance to re-ionization and the possibility they offer of tracing the contribution by individual polluters, such as single supernovae (SNe).

In his talk, K. Schlaufman made a distinction between stars belonging to population III.1 (really first stars) and III.2 (star that formed from material with no metals but within the UV radiation from III.1 stars). In any case, he underlined that the very 
first stars are expected in very biased environments, such as possibly the core of giant ellipticals. In the local environment (Milky Way and its neighbours), the most biased ambient is the bulge. This is the place where the very first stars should be looked. I notice that in order to properly design the searches for the very "first stars", we should understand how many "first stars" do we really expect. Answering this question requires a dedicated study.

There were several very interesting talks on extremely metal-poor stars, stemming from the nice review by A. Frebel. Various contributions concerned observations (W. Aoki, D. Yong, H. Li, T. Hansen, K. Venn, V. Hill), but there were also suggestive talks on models (C. Kobayashi, D. Romano, E. Starkenburg). Several authors remarked that we are finally reaching the limit of what can be observed. Keller et al. (2014) documented a star with $[\mathrm{Fe} / \mathrm{H}]<-7$ that is actually an upper limit because no iron line was detected in the spectrum. This prompted the question about what is the limit that can be measured? As shown in the conference, the technical limits are $[\mathrm{Ca} / \mathrm{H}]=-9.4,[\mathrm{Fe} / \mathrm{H}]=-7.2,[\mathrm{Mg} / \mathrm{H}]=-6$, and we are now close to these limits.

Most attention during the symposium was focused on the carbon enhanced metal-poor stars with no enhancement of the s-process nuclei (the so-called CEMP-No star). Some of them are the most Fe-poor objects known. For this reason these objects are considered the best proxy for the "first stars". However, as a person quite far from the field I have a somewhat different point of view: in fact, I remark that metal-poor C-rich stars are not that metal-poor in terms of overall metal content Z. Rather, their composition indicates that the production of $\mathrm{C}$ and Fe are decoupled at low metallicity. However, I notice that this happens also at high metallicity. Finally I notice that also the production of $\mathrm{Ba}$ (and possibly $\mathrm{Sr}$ too) is decoupled from that of Fe at low metallicity.

\section{Globular clusters}

Globular clusters (GCs) are fundamental tracers of the halo. At the meeting there were nice observational reviews updating on the most recent investigations concerning both Milky Way (E. Carretta, G. Piotto, G. Da Costa, D. Geisler, A. Milone) and external galaxies (S. Larsen, M. Hilker, J. Brodie). These talks showed that there is a strong synergy between (UV) photometry and spectroscopy in understanding and quantifying the overall pattern. There were also interesting theoretical contributions by C. Charbonnel and O. Gnedin. Summarizing, it is now clear that (all?) GCs host multiple stellar populations with significant differences in $\mathrm{He}, \mathrm{CNO}, \mathrm{Na}, \mathrm{Mg}, \mathrm{Al}$, and sometimes $\mathrm{Si}$ and $\mathrm{K}$ abundances. Most likely, stars in GCs show discrete or at least clumpy distributions in the abundances of these elements. Simplifying, there are two main populations of stars in GCs: those with a composition indistinguishable from that of field halo stars and those that are enriched in $\mathrm{Na}$ and $\mathrm{Al}$, and depleted in $\mathrm{O}$ and $\mathrm{Mg}$. The first group is thought to have the original composition from which the GC formed, and are then called First Generation (FG) stars, while the second group is found to have their composition altered by H-burning at high temperature in as yet not clearly identified group of FG stars, and is then thought to have formed later as a second generation (SG). SG stars make up about $2 / 3$ of the stars of GCs, but are rare in the general field.

For what concern their relation with the halo, it was remarked that GCs now make up some $1.2 \%$ of the halo, but they were surely more massive in the past. A conservative estimate (based on the fraction of SG stars found in the field, about $2.5 \%$, and on the assumption that they were all lost from GCs) is that $\sim 5 \%$ of the halo stars comes from GCs. However, it was commented that this might represent a substantial underestimate of the contribution of GCs to the formation of the halo that maybe even as large as $50 \%$ 
if FG had indeed initially 10 more mass then SG as given by most scenarios for multiple populations (only a fraction of the FG stars likely produced the material from which the SG stars formed).

Many points in this overall scenario are still not well understood. First of all, how GCs formed? Many cosmologists think of pre-galactic formation (at least for blue sequence GCs) linking them to reionization. However, no GC star so far was found with $Z<$ $0.003 Z_{\odot}$. In his talk, Gnedin remarked that the formation of GCs seems to be related to gas-rich mergers. The case of the Antennae galaxies, where many very massive young stars clusters are observed, may support this view. Another interesting issue is the presence of two sequences of GCs (blue and red GCs) in a large fraction of the external galaxies. How to produce these blue and red sequences? Most authors explain the presence of these sequences as a consequence of a given merging history: blue, metal-poor GCs are generally related to the halo or dry mergers, while red, metal-rich clusters are thought to be related to the bulge or thick disk, and in general to gas-rich mergers. If this scenario is correct, why the presence of two sequences is so common? We might indeed expect a wide range of formation histories leading to a correspondent range in the properties of the GC systems. J. Brodie discussed this point in her talk and proposed a general twophase formation scheme for galaxies. This is very intriguing; in my opinion we should also understand why the specific frequency of GCs in galaxies and in different populations in a given galaxy have such large variations as observed.

In addition, it seems that there is a relation between at least some GCs and the nuclear clusters (see the talk by G. Da Costa). On turn, this relates to how nuclear clusters form (in situ or migrated) and on what is the relation between the nuclear star clusters and (super?)-massive BHs in the central regions of galaxies. Of course, it is possible that GCs are only related to nuclear clusters in dwarf galaxies, however this point certainly merits further investigations.

Though this symposium was devoted to the halo, I think it worth opening here a parenthesis on the bulge. Gnedin suggested that possibly a single large late gas-rich merger have produced the metal-rich GCs in the Milky Way. Since the metal-rich GCs in the MW are mainly in the bulge, is this indicating a merger origin for the bulge? This looks quite different from what Gerhard said in his talk: "The bulge/bar formed from perturbation of the (thick?) disk with little or no evidence for a previous bulge". Is there a tension between these two points of view? Still about the bulge: not mentioned during the meeting is the evidence that the bulge is old from the colour-magnitude diagram (Zoccali et al. 2003).

Summarizing, most have still to be understood about the early evolution of GCs. The observations (Carretta, Piotto, Milone) indicate that once they formed they had a complex evolution. C. Charbonnel observed that powerful explosions are required to empty of gas young massive clusters (observed) and GCs (presumed from lack of contribution by SNe to next generations). I note here that GCs are so massive that the high end of the stellar initial mass function is very well sampled. This leaves ample room for the formation of stars of very high mass, the most probable precursors of such powerful explosions.

\section{Dwarf spheroidals}

Dwarf spheroidals are another major constituents of the (outer) galactic halo. Many new dSph's have been discovered recently both in the Milky Way and in nearby galaxies. The talk by J. Simon presented an updated census and discussed the main parameters of these clusters. G. Battaglia discussed the very hot issue of the structure of the dSphs: are 
they cusped (as predicted for simple dark matter structures) or rather have isothermal cores (as possible if baryon feedback play a very important role)? She discussed how the impact of anisotropy should be properly understood before any conclusion can be drawn. Her conclusion is that at the current status cores seem favoured. This is likely very important for an understanding of the missing satellite issue because cores are more easily destroyed than cusps (talks by Gerhard and Simon).

V. Hill showed that metal-rich populations tend to be more centrally concentrated in dSph's. This suggested to me the question if some of the dSph have formed itself from accretion of smaller fragments. Personally, I think that good candidates for a similar scenario are Sagittarius and Fornax dSph's, not only because they are larger, but also because they host several GCs that might be the signature of a dramatic past (see previous Section). Indeed the Sagittarius dSph seem to have been in origin a mediumsized galaxy (original $M_{V} \sim-15$ : A. Ferguson). Also the history of Carina dSph seem to include accretion (Hill).

The chemical composition of dSph's was also discussed. Metal-rich stars in dSph's have a composition clearly different from the bulk of the (inner) halo; they are also clearly too young (V. Hill). There is a wide diversity of dSph's but yet scaling relations are valid. The colour-magnitude diagrams allow to derive the star formation histories, showing sometimes evidence for different star formation episodes. On the whole, however, there is a tight $[\mathrm{Fe} / \mathrm{H}]_{\text {bulk }}-L / L_{\odot}$ relation (Kirby et al. 2013). The internal history of metal-enrichment shows that the decrease in [alpha/Fe] ratio does not occur at the same $[\mathrm{Fe} / \mathrm{H}]$ for all dSph's: the knee follows the luminosity (V. Hill). While there is almost no dispersion in the metal-to-metal abundance ratios at high metallicity $([\mathrm{Fe} / \mathrm{H}]>-2)$ for the massive $\mathrm{dSph}$, there is for small ones.

K. Venn discussed the increased evidence for the presence of extremely metal-poor stars in dSph's. She showed that the dispersion in heavy elements, e.g. Ba, is huge al low metallicity. Given the small mass of the dSph's considered in the study she presented, this can be attributed to the fact that we are sampling the enrichment of a single SN in each case. In a few cases very low $\mathrm{Mg}$ abundances are found; they are interpreted as due to enrichment only from SNIa (Fe-enriched from SNIa pockets). In addition, J. Simon presented the discovery of CEMP-No stars in dSph. D. Romano discussed modelling of these results. The chemical composition of dSph's stars requires the presence of large outflows, but these outflows maybe Fe-rich (Recchi et al. 2001). The contribution by single SNe may explain outliers.

\section{What's next}

First I notice that there was much more at this meeting. P. Schechter provocatively asked why are galaxies on a 2-D fundamental plane while DM halos are on a 1-D? J. Knapan presented cases of truncation in face-on galaxies. J. Penarrubia presented a new approach to the study of dynamics that he called "gravitational mechanics", based on dynamical invariants, introducing Gaussians diffusion coefficients. B. Elmegreen discussed the accretion vs star formation in dwarf galaxies. L. Greggio presented the case for studies of stellar populations with future instruments (ELT, JWST).

\subsection{The halo and the disk}

There are then a few points not discussed in the Symposium that personally found of interest. For instance, it is well known that the Milky Way disk metallicity did not change significantly in the last $\sim 10 \mathrm{Gyr}$; this implies that the gas fraction stayed roughly constant at about $10 \%$. But gas is transformed into stars at about $1 \mathrm{M}_{\odot} / y r$ rate. On the other hand, there is strong evidence that the Milky Way has not experienced major 
mergers in the last $\sim 8-10$ Gyr. This requires continuous infall of gas at roughly this rate: about $10^{10} M_{\odot}$ of gas should have been accreted by the disk during this epoch. Most of this gas must have been in the form of small clouds. From where all this gas came?

\subsection{The Magellanic Clouds}

I also found rather surprising that there was almost no mention during the Symposium of the fact that by far the two most conspicuous objects in the Milky Way halo are the Magellanic Clouds: in total, they contain $\sim 10^{10} M_{\odot}$ of baryonic matter, that is more than 10 times the rest of the halo. Though they are probably losing gas (possibly contributing to the infall in our galaxy) they are still gas-rich objects. This possibly indicates that they were not so close to the MW up to a quite recent past. However, I expect the destiny of the Magellanic Clouds is to be accreted by the MW on a Gyr or less scale. This will be a significant merger event, and will possibly change completely the MW halo.

\subsection{List of additional questions}

Finally, what about the (thick) disk/halo interface and the origin of the stellar disk? Was there a smooth transition between early accretion and disk formation (what I call a dissipative component)? Was not there a late bombardment phase? Are dry mergers a good approximation for the early formation of the halo (M. Hirschman)? Why most satellites are on well defined planes (J. Simon, A. Helmi)? What instruments for the future (L. Greggio)?

Summarizing, this meeting was of exceedingly interest, but the potential of development of or knowledge about the halo of our Galaxy is far from been exhausted. In the next few years we expect dramatic progresses from the new spectroscopic survey such as APOGEE, LAMOST, GES, and moreover from the Gaia mission. Also modelling may progress thanks to a better understanding of the feedback mechanisms in galaxy formation and evolution. We expect then a revolution in the field and we should be ready to accept new ideas and scenarios. So, good work to every one!

\section{References}

Ibata, R.A., Lewis, G.F., McConnachie, A.W., Martin, N.F., Irwin, M.J., et al. 2014 ApJ 780, 128

Keller, S. C., Bessell, M. S., Frebel, A., Casey, A. R., Asplund, M., et al. 2014, Nature, 506, 463

Kirby, E. N., Cohen, J. G., Guhathakurta, P., Cheng, L., Bullock, J. S., \& Gallazzi, A. 2013, ApJ, 779, 102

Mackey, A. D., Huxor, A. P., Ferguson, A. M. N., Irwin, M. J., Tanvir, N. R., et al. 2010, MNRAS, 401, 533

Recchi, S., Matteucci, F., \& D'Ercole, A, 2001, MNRAS, 322, 800

Tissera, P. B., White, S. D. M., \& Scannapieco, C. 2012, MNRAS, 420, 255

Tissera, P. B., Scannapieco, C., Beers, T. C., \& Carollo, D. 2013, MNRAS, 432, 3391

Tollerud, E. J., Bullock, J. S., Strigart, L. E., \& Willman, B. 2008, ApJ 688, 277

Zoccali, M., Renzini, A., Ortolani, S., Greggio, L., Saviane, I., Cassisi, S., et al. 2003, A\&A, 399, 931

Zolotov, A., Willman, B., Brooks, A. M., Governato, F., Brook, C. B., et al. 2009, ApJ, 702, 1058

Zolotov, A., Willman, B., Brooks, A. M., Governato, F., Hogg, D. W., et al. 2010, ApJ, 721, 738 